\title{
Título
}

\section{Estigma: la relevancia del contacto social en el trastorno mental.}

Stigma: the relevance of social contact in mental disorder.

\section{Resumen}

Introducción: El estigma asociado a la enfermedad mental es un problema de salud, discriminando y limitando las oportunidades de las personas que lo padecen. El contacto social con personas que sufren un trastorno mental es una estrategia utilizada para producir cambios en los estereotipos en la población. El objetivo del estudio fue examinar las diferencias en el nivel de estigma en muestras con contacto social y población general.

Metodología: El estudio incluye dos experiencias. La primera $(n=42)$ incluye jugadores de una liga de futbol abierta en la que juega un equipo de jugadores con diagnóstico de esquizofrenia. En la segunda se compara una muestra sin contacto conocido $(n=62)$ y una muestra con contacto $(n=100)$. La herramienta utilizada de evaluación fue el AQ27, en versión española (AQ-27-E). Se analizaron la diferencia de medias entre las dos muestras, de cada una de las 9 subescalas.

Resultados: En la primera experiencia, todas las subescalas presentaron menor puntuación en post-contacto que en pre-contacto, excepto Responsabilidad, dos subescalas que mostraron diferencias significativas fueron Coacción $(\mathrm{t}=6,057, \mathrm{p}=$ $0,000)$ y Piedad $(\mathrm{t}=3,661, \mathrm{p}=0,001)$.

En la segunda experiencia, siete subescalas mostraron nivel de significancia $(\mathrm{p}=<0,05)$. Responsabilidad y Segregación no lo mostraron.

Conclusiones: Se observa que el contacto social realizado en entornos cotidianos puede producir un impacto positivo en la reducción del estigma, esto puede contribuir a favorecer la igualdad de oportunidades. 
Palabras clave: Estigma, contacto social, enfermedad mental, trastorno mental, esquizofrenia, programas comunitarios.

Introduction: The stigma associated with mental illness is a health problem, discriminating and limiting the opportunities for people who suffer. Social contact with people suffering a mental disorder is a strategy used to produce changes in the population stereotypes. The aim of the study was to examine differences in the level of stigma in samples with social contact and population.

Methodology: The study includes two experiences. The first $(n=42)$ includes a league players open playing football on play a team of players with schizophrenia. In the second known contact a sample without $(n=62)$ and a sample with contact $(n=100)$ is compared. The evaluation tool used was the AQ-27, Spanish version (AQ-27-E). The mean difference between the two samples, each of the 9 subscales were analyzed.

Results: In the first experimence, all subscales had lower scores on post-contact in precontact, except Responsibility, two subscales that showed significant differences were Duress $(\mathrm{t}=6.057, \mathrm{p}=0.000)$ and Mercy $(\mathrm{t}=3.661, \mathrm{p}=0.001)$. In the second experimence, seven subscales showed significance level $(\mathrm{p}=<0.05)$. Segregation responsibility and did not show it.

Conclusions: It is observed that the social contact carried out in daily environments can have a positive impact on the reduction of stigma, this can contribute to favor equality of opportunity.

Key words: Stigma, social contact, mental illness, mental disorder, schizophrenia, community programs.

\section{Introducción}


Las personas que sufren una enfermedad mental son más severamente estigmatizadas que aquellas que poseen otras condiciones médicas, sociales, políticas o económicas ${ }^{1-3}$. Así mismo, miembros de la población en general son menos propensos a interactuar con personas relacionadas con las enfermedades mentales ${ }^{4}$. En este sentido las actitudes de los profesionales de la salud en general y de la enfermería en particular son de suma importancia ya que constituyen un factor determinante en la calidad de la atención prestada a las personas con enfermedad mental ${ }^{5}$. Las actitudes estigmatizantes contribuyen a discriminar en situaciones de la vida cotidiana, restringiendo las oportunidades. Como consecuencia y por miedo a la estigmatización, los individuos con enfermedad mental pueden aislarse de la sociedad, con el consiguiente empeoramiento de sus condiciones clínicas y su pronóstico ${ }^{6-8}$.

Además de las experiencias de discriminación directa de otras personas, las personas con enfermedades mentales pueden ser perjudicadas por discriminación estructural, como una menor inversión de recursos de atención de la salud ${ }^{9-10}$. Así mismo, las personas con trastornos mentales también suelen experimentar un trato desigual en las condiciones de salud física, lo que podría contribuir al aumento de la morbilidad y la mortalidad prematura ${ }^{11-12}$.

La estigmatización puede conceptualizarse como un proceso que implica complejas interacciones cognitivo-conductuales entre el individuo y el entorno social ${ }^{13}$. En el estigma concurren diversos componentes, los estereotipos, la separación, la pérdida de estatus y la discriminación, produciéndose estigmatización cuando se ejerce desde una posición de poder ${ }^{14}$, por este motivo el estigma denota un descrédito y una marca de deshonra que generalmente conduce a un comportamiento negativo en su portador.

Aunque el concepto de estigma es fundamental para comprender la experiencia de exclusión social, no la explica en su totalidad ya que no identifica las acciones necesarias para promover la inclusión social. En el estigma se han identificado tres problemas que están interrelacionados: el problema del conocimiento (la ignorancia), el problema con las actitudes (el prejuicio) y el problema con el comportamiento (la discriminación) ${ }^{15-17}$. Para dar respuesta a estos problemas relacionados con el estigma en salud mental se han desarrollado diferentes enfoques teóricos, incluyendo modelos cognitivo sociales ${ }^{18}$ en los que se dan relevancia a los estereotipos (creencias negativas acerca de un grupo), el prejuicio (reacciones emocionales negativas como el miedo y/o 
la ira, de acuerdo con las creencias estereotipadas), y la discriminación (exclusión de las oportunidades sociales y económicas a consecuencia del comportamiento de los prejuicios).

Se han ha llevado a cabo estudios sobre las estrategias que se deben realizar para producir cambios en los estereotipos y se han identificado tres enfoques para el cambio de las actitudes estereotipadas: la educación, a fin de reemplazar las actitudes estigmatizantes con concepciones precisas acerca del trastorno; el contacto, que busca el cambio a través de la interrelación directa con personas con un trastorno; y la protesta, que busca suprimir actitudes estigmatizantes hacia personas con trastorno mental ${ }^{18}$. Hasta hace muy poco, estos estudios han evaluado el cambio de conocimiento o actitud, o ambos, pero no han evaluado el impacto en el comportamiento. En un meta análisis de Corrigan y colaboradores sobre intervenciones para abordar el estigma público concluyeron que el contacto directo era superior al contacto filmado y, para los adultos, el contacto era más eficaz que la educación ${ }^{20}$.

Como hemos dicho, una de las vías para el cambio de actitudes de la población hacia la enfermedad mental está en favorecer la interacción entre personas con trastorno mental y la población general, aunque también se reconoce que son necesarias más investigaciones en este campo para determinar los efectos de este contacto ${ }^{21}$. En la actualidad algunas revisiones sistemáticas apoyan la idea de que el contacto social es la intervención más efectiva para adultos ${ }^{22}$. El objetivo de este estudio fue examinar las diferencias en el estigma entre la población en general y población con contacto social con personas con trastorno mental. Para ello, se analizaron muestras paralelas en dos experiencias comunitarias diferentes.

\section{Metodología}

Se han realizado dos experiencias, la primera con un diseño pre-post contacto y la segunda con un corte transversal con dos muestras diferentes. La herramienta utilizada de evaluación del estigma fue el AQ-27, en su versión española (AQ-27-E). El Attribution Questionaire, fue creado inicialmente por Weiner et al. (1988), realizándose diferentes revisiones. Corrigan, Watson, Warpinski y Gracia (2004) incrementaron el número de ítems hasta 27, configurando el Attribution Questionaire $27(A Q-27)^{23-25}$, 
que finalmente se ha utilizado en las dos experiencias en su versión traducida al idioma español por Muñoz M, Guillén, Pérez Santos y Corrigan ${ }^{24}$.

La primera experiencia consistió en favorecer el contacto social a través de la participación de un equipo de futbol sala, formado por 11 jugadores con diagnóstico de esquizofrenia, en un campeonato abierto para toda la población. El equipo asignó un capitán, responsable de realizar la inscripción y asistir a todas las reuniones de coordinación que convocó la dirección del campeonato. Así mismo, este capitán era el responsable de transmitir la información en ambas direcciones: desde la dirección del torneo a los integrantes del equipo, y desde los integrantes del equipo a la coordinación del campeonato.

La primera valoración (pre) se realizó una vez iniciado el campeonato: a tal efecto, se solicitó a los integrantes de los otros equipos que respondiesen al cuestionario AQ-27-E. Para reclutar la muestra se organizó una reunión con un representante de cada equipo donde se les informó del estudio de valoración de la percepción sobre la salud mental, invitándoles a participar en el mismo y a hacer extensiva la invitación al resto del equipo. A esta primera valoración respondieron 63 individuos.

Al finalizar el campeonato, cuatro meses más tarde, a los jugadores que habían respondido a la valoración pre se les solicitó que lo hiciesen una segunda vez (post). Previamente se les informó que habían estado jugando con un equipo formado por personas con enfermedad mental. A esta segunda pasación respondieron 42 individuos, siendo esta la muestra definitiva del estudio $(\mathrm{N}=42)$.

En la segundo experiencia se valoró el contacto social a través de la convivencia cotidiana, se realizó con dos muestras independientes. La primera se obtuvo solicitando a población general la cumplimentación del cuestionario AQ-27-E. En esta primera muestra buscábamos la opinión de la población general independientemente del grado de conocimiento o desconocimiento que tuvieran en relación con el trastorno mental. Respondieron al cuestionario 64 individuos $(\mathrm{N}=64)$. La segunda muestra se obtuvo de personas que acudían a un centro cívico. En este centro cívico compartían espacios comunes con personas con enfermedad mental, ya que en este se realizaban actividades. A los usuarios del centro cívico se les solicitó la cumplimentación del cuestionario AQ27-E. Contestaron 100 individuos, $(\mathrm{N}=100)$. 
En ambas experiencias, la participación fue voluntaria, requiriendo previamente la firma del correspondiente consentimiento informado. Como criterios de exclusión se formularon ser integrante del equipo con personas con TM, no entender el idioma españolo catalán y ser menor de 18 años.

Las dos experiencias se realizaron en el municipio de Sta. Coloma de Gramenet, población de 119.182 habitantes, situado en la provincia de Barcelona. En esta localidad existe desde el año 1930 un hospital monográfico de salud mental. En la actualidad, además de unidades de hospitalización psiquiátrica la localidad cuenta con servicios de atención comunitaria integrados en la red de atención pública a la salud mental.

Las personas con trastorno mental que forman parte del equipo de futbol estaban vinculadas al sistema sanitario público, acudiendo regularmente al centro de rehabilitación comunitaria y visitándose periódicamente en el Centro de Salud Mental de Adultos (CSMA).

La aprobación ética fue concedida por el Comité Ético de Investigación Clínica del Parc de Salut Mar de Barcelona en Febrero del 2015. La recogida de datos se realizó entre Marzo y Julio de 2015.

\section{Instrumentos de medida}

La escala AQ-27-E está basada en la teoría de la atribución incluyendo el modelo de responsabilidad como una parte de explicación del estigma. El modelo de responsabilidad identifica dos vías de funcionamiento. Cuando se atribuye a la persona responsabilidad sobre la enfermedad o que puede ejercer cierto control sobre ella, esta percepción facilita actitudes de enojo con comportamientos de control, castigo o represión. Si se considera a la persona como una víctima del trastorno o se considera que el trastorno es incontrolable, las actitudes principales serán de piedad, y los comportamientos más frecuentes serán de ayuda ${ }^{18,25}$.

Corrigan $^{18}$ también observó comportamientos relacionados con la percepción de peligrosidad agregando un segundo modelo. Con ello la atribución de conductas peligrosas a una persona conduce a la emoción del miedo, que a su vez, conduce a la aprensión o al comportamiento evasivo. 
En 2004, Corrigan, Watson, Warpinski y Gracia $^{25}$ determinaron el número de ítems a 27 (AQ-27) y el número de factores a nueve: responsabilidad, piedad, cólera, peligro, miedo, ayuda, coerción, segregación y evitación. El cuestionario AQ-27-E es un instrumento de medida autoadministrado diseñado para valorar actitudes y comportamientos relacionados con la enfermedad mental: incluye un caso que representa un hipotético personaje (José) que sufre una severa enfermedad mental.

El cuestionario está basado en los dos modelos explicativos del estigma: la teoría de la atribución y la teoría de la peligrosidad ${ }^{25,26}$. Recoge 27 ítems que se estructuran en las 9 subescalas relacionadas arriba, de 3 ítems cada una. Los 27 ítems de los que consta se evalúan en un formato tipo Likert de 9 puntos, que van desde No en absoluto (1 punto) a Mucho (9 puntos). La persona evaluada responde las 27 preguntas señalando con un círculo la respuesta que mejor refleje su opinión sobre cada una de las frases. El tiempo de administración es de 10 - 20 minutos. El resultado de cada una de las 9 subescalas se obtiene con el sumatorio de los 3 ítems correspondientes; el rango observable en la puntación de las subescalas oscila entre 3 y 27.

El cuestionario AQ-27, ha sido traducido y validado al idioma español por Muñoz M et al. En el estudio de validación se evaluó la fiabilidad del AQ-27-E, mostrando un alfa de Crombach de 0,855 para el total de la escala. Así mismo presentó adecuadas propiedades psicométricas para la evaluación del estigma en población de habla española ${ }^{25}$.

Para el estudio de las variables sociodemográficas se incluyó también una ficha de preguntas básicas que recogía datos de edad, género y nivel de escolarización.

\section{Análisis estadístico}

Las variables sociodemográficas se estudiaron mediante estadísticos descriptivos.

Para el análisis del estudio se analizó la diferencia de medias, en las dos experiencias, de cada una de la subescalas de AQ-27-E, entre pre y post-contacto en la actividad deportiva, población general y con contacto con personas con trastorno mental en la convivencia cotidiana. Para este análisis de medias se utilizó la t-test para muestras dependientes en la primera experiencia y para muestras independientes en la segunda. 


\section{Resultados}

En la experiencia del campeonato de futbol la muestra fue de 42 individuos ( $\mathrm{N}=42)$. El $100 \%$ fueron hombres, con una media de edad de 33 años $(\mathrm{DE}=10,38)$ y un rango de 19 a 64 años. El nivel de estudios se situaba mayoritariamente en secundarios $(63,4 \%)$ seguido de universitarios $(22 \%)$.

Realizada la comparación de medias de las subescalas AQ-27-E, las dos que presentaron mayor variación fueron Coacción, con una disminución de 4,33 puntos, y Piedad con una disminución de 2,71 puntos entre la primera pasación (pre) y la segunda (post). Estas dos subescalas fueron las únicas que mostraron diferencias significativas: Coacción $(\mathrm{t}=6,057, \mathrm{p}=0,000)$ y Piedad $(\mathrm{t}=3,661, \mathrm{p}=0,001)$ (Tabla 1$)$.

En general las medias de todas las subescalas presentaron menor puntuación en post que en pre, excepto Responsabilidad, que presentó un incremento de 0,85 puntos y Evitación, que presentó un incremento de 1,28 puntos, aunque en esta subescala la lectura del resultado debe invertirse. En Ayuda prácticamente se mantuvo igual, con un ligero incremento de 0,05 puntos (Tabla 1 ).

En la segunda experiencia, la muestra de población general presentó una distribución por género en el que las mujeres eran mayoritarias $(62,1 \%)$. La media de edad fue de 44 años $(\mathrm{DE}=15,13)$ con un rango de 19 a 77 años. El nivel de estudios se situó mayoritariamente en primarios (36,5\%) seguido de secundarios (34,9\%).En la muestra en la que sí habían tenido contacto con personas con enfermedad mental, las mujeres representaron el 69\%, con una media de edad de 55 años $(\mathrm{DE}=18,59)$ con un rango de 19 a 87 años. El nivel de estudios se situó mayoritariamente en primarios $(58,7 \%)$ seguido de secundarios $(25 \%)$.

En la comparación de medias de las subescalas de AQ-27-E, seis de ellas (Responsabilidad, Ira, Peligrosidad, Miedo, Coacción y Segregación) presentaron puntuaciones más bajas en el grupo con contacto respecto al grupo de población general (Tabla 2). En Piedad, Ayuda y Evitación presentaron, en cambio, mayor puntuación. Las cuatro subescalas que mostraron mayor diferencia fueron: Ayuda con una diferencia de 3,68 puntos, Peligrosidad con una diferencia de 3,00 puntos, Evitación con un diferencia de 2,85 puntos (se debe leer inversamente) y Miedo con una diferencia de 
2,68 puntos. De las nueve subescalas, siete mostraron nivel de significancia $(\mathrm{p}=<0,05)$. Por el contrario, Responsabilidad y Segregación no lo mostraron.

\section{Discusión}

El contacto social puede producir mejoras en las actitudes de la población en relación a las personas que sufren un trastorno mental. En la segunda experiencia la comparación de las dos muestras sugiere que el contacto a través de una relación cotidiana permite a las personas aprender a actuar, sentir y pensar positivamente en relación al trastorno mental. Esta actitud positiva favorece un mayor control sobre las diversas situaciones que se pueden plantear cuando se establece una relación con personas con una enfermedad mental, repercutiendo positivamente en estas ${ }^{27}$. Los resultados de la presente investigación están en línea con otros estudios anteriores, que mostraron que las actitudes se pueden cambiar a través del contacto social ${ }^{27-29}$ observándose mayor impacto cuando este contacto se produce a través de la relación cotidiana y durante un tiempo prolongado.

Se debe señalar que en la actividad deportiva programada si bien se produjeron cambios positivos en todas las dimensiones, estos cambios fueron significativos en Piedad y Segregación; es decir, las personas que habían intervenido en la actividad de futbol cuando tuvieron conocimiento de la participación de un equipo formado por personas con trastorno mental parecen mostrar una mejora en la actitud en estas dos dimensiones. Asimismo, en relación a la segunda experiencia, también se observaron diferencias positivas en todas las dimensiones, siendo estas diferencias significativas en un número mayor de dimensiones Piedad, Ira, Peligrosidad, Miedo, Ayuda, Coacción y Evitación, esto sugiere una actitud más positiva en las personas si estas conviven de forma espontánea en actividades cotidianas sin tener éstas porqué ser organizadas ${ }^{30}$. Por otra parte y ajustándonos a los modelos de atribución y peligrosidad se podría deducir que en la segunda experiencia, se observaron diferencias en la visión sobre enfermo mental, considerándolo más como una víctima que como responsable de su enfermedad, así mismo también se produjo una menor percepción de la peligrosidad ${ }^{25,26}$.

Como se observa el número de dimensiones en las que se aprecian diferencias significativas fue mayor en la segunda experiencia con respecto a la primera. Esto 
podría tener su explicación en el hecho de que en la primera, aunque los participantes eran conocedores de que habían convivido con personas con trastorno mental no los llegaron a identificar; en cambio, en la segunda, los individuos que participaron sí que conocían a estas personas, esto pone de manifiesto la importancia que tiene no solo el contacto sino también la relación personal y social entre los individuos. Lo que nos llevaría a la necesidad de realizar una reflexión sobre el impacto de la calidad del contacto en la modificación de actitudes estigmatizantes. A su vez, se pone de relevancia la importancia que parece tener el tiempo de contacto habitual y prolongado que se produjo en el segundo estudio frente al contacto esporádico del primer estudio en la mejora de la actitud frente a las personas con trastorno mental.

Así mismo, en esta comparación de resultados entre las dos experiencias, se observa que dos dimensiones se comportan de diferente forma. Por un lado, Piedad mostró una disminución en la actividad deportiva y en cambio mostró una puntuación más alta en la muestra de contacto del segundo estudio. A su vez, Ayuda, que no cambió en la intervención del futbol, mostró una puntuación alta en la muestra con contacto continuado de la segunda experiencia. Como hemos referido anteriormente, una explicación la podríamos situar en el modelo de atribución, adjudicando mayor responsabilidad sobre la enfermedad a la persona con trastorno mental en el primer estudio y apareciendo como víctima en el segundo ${ }^{25,26}$. Por lo que se puede observar la relación cotidiana y de mayor duración correspondiente a la segunda experiencia, establece relaciones personales más próximas que conducen a una mayor implicación en las circunstancias personales ajenas.

Por su parte, las dimensiones que representan la esfera de la percepción de peligro (peligrosidad, miedo y evitación) puntúan más baja en las muestras con contacto en ambos estudios. Esta importante disminución en la esfera de la percepción de peligro se podría explicar también por la relación más cotidiana y de mayor duración; podría deducirse que el mayor conocimiento de la persona ayudaría a cambiar positivamente ideas preconcebidas, reduciendo los miedos y la sensación de peligro.

Por último debemos señalar que en relación a la experiencia del campeonato de fútbol, se explicó a los participantes en el torneo de futbol que habían estado compitiendo con un equipo con personas que presentaban trastorno mental, aunque como hemos señalado 
anteriormente consideramos la información aportada suficiente debemos señalar como limitación la no identificación de las personas con trastorno mental debido a la negativa de estas. Así mismo, este aspecto lo debemos relacionar con el concepto de autoestigma que pueden sufrir las personas con un trastorno mental ${ }^{31}$. Por otro lado se debe de tener en cuenta la variable del género en el estudio del futbol ya que es este caso todos los participante fueron hombres pudiendo este factor influir en la atribución de responsabilidad delante de la enfermedad.

\section{Conclusiones}

De los dos estudios realizados y con los resultados obtenidos se puede observar que el contacto social recoge un menor estigma sobre las personas con trastorno mental en la población. Además, el contacto habitual y prolongado con personas que presentan un trastorno mental parece producir menor estigma que el contacto puntual y limitado en el tiempo.

Así mismo, observamos que el contacto social realizado en entornos utilizados por la población general (deportivos, culturales) produce impacto positivo en la reducción del estigma, y además pensamos que el situar al mismo nivel a personas con trastorno mental y población en general, favorece también la igualdad de oportunidades. Por ello sugerimos la importancia de establecer estrategias colaborativa entre la comunidad y los profesionales de enfermería, en el compromiso de establecer planes de acción y trabajo colaborativo que contribuyan a la reducción del estigma.

Es necesario continuar profundizando en el conocimiento sobre la reducción del estigma que rodea a la enfermedad mental, realización de nuevos estudios que investiguen estrategias que se basen en el contacto social en ámbitos comunitarios abiertos a la población en general introduciendo la variable de la calidad del contacto como factor para la reflexión. 


\section{Referencias}

1. Marwaha S, Johnson S (2005) Views and experiences of employment among people with psychosis: a qualitative descriptive study. Int J Soc Psychiatry $51: 302-316$.

2. Baumann A (2007) Stigmatization, social distance and exclusion because of mental illness: the individual with mental illness as a 'stranger'. Int Rev Psychiatry 19:131-135.

3. El-Badri S, Mellsop G (2007) Stigma and quality of life as experienced by people with mental illness. Australas Psychiatry15:195-200.

4. Halter M (2008) Perceived characteristics of psychiatric nurses: stigma by association. Arch Psychiatr Nurs 22:20-26.

5. Kassam A, Glozier N, Leese M, Henderson C, Thornicroft G. Development and responsiveness of a scale to measure clinicians' attitudes to people with mental illness. Acta Psychiatr Scand. 2010;122:153-61.

6. Vauth R, Kleim B, Wirtz M, Corrigan P (2007) Self-efficacy and empowerment as outcomes of self-stigmatizing and coping in schizophrenia. Psychiatry Res 150:71-80.

7. Yap MB, Wright A, Jorm AF (2010) The influence of stigma on young people's help-seeking intentions and beliefs about the helpfulness of various sources of help. Soc Psychiatry Psychiatr Epidemiol.

8. Mojtabai R (2010) Mental illness stigma and willingness to seek mental health care in the European Union. Soc Psychiatry Psychiatr Epidemiol 45(7):705-712.

9. Corrigan PW, Markowitz FE, Watson AC. Structural levels of mental illness stigma and discrimination. Schizophr Bull 2004; 30: 481-91.

10. Link BG, Phelan JC. On stigma and its public health implications. Stigma and global health: developing a research agenda; Bethesda, Maryland, USA; Sept 57, 2001.

11. Thornicroft G. Premature death among people with mental illness. BMJ 2013; 346: $\mathrm{f} 2969$.

12. Thornicroft G. Physical health disparities and mental illness: the scandal of premature mortality. Br J Psychiatry 2011; 199: 441-42.

13. Norman RM, Sorrentino RM, Gawronski B, Szeto AC, Ye Y, Windell D (2010) Attitudes and physical distance to an individual with schizophrenia: the 
moderating effect of self-transcendent values. Soc Psychiatry Psychiatr Epidemiol 45(7):751-758.

14. Link BG, Phelan JC. Conceptualizing stigma. Annual Review of Sociology. 2001; 27:363-385.

15. Thornicroft G, Brohan E, Kassam A, Lewis-Holmes E. Reducing stigma and discrimination: Candidate interventions. International Journal of Mental Health Systems. 2008; 2:3.

16. Thornicroft G, Rose, D, Kassam, A, and Sartorius, N. Stigma: ignorance, prejudice or discrimination?. Br J Psychiatry. 2007; 190: 192-193.

17. Thornicroft G: Shunned: Discrimination Against People With Mental Illness. Oxford: Oxford University Press; 2006.

18. Corrigan PW. Mental health stigma as social attribution: implications for research methods and attitude change. Clin Psychol Sci Pract. 2000;7: 48-67.

19. Thornicroft G, Mehta N, Clement S, Evans-Lacko S, Doherty M, Rose D, et al. Evidence for effective interventions to reduce mental-health-related stigma and discrimination. Lancet. 2016; 358: 2110-2112.

20. Yang L, Cho SH, Kleinman A. Stigma of Mental Illness. In: Patel V, ed. Mental and neurological public health: a global perspective: Elsevier, 2010.

21. Corrigan PW. River LP. Lundin RK, et al. Three strategies for changing attributions about severe mental illness. Schizophr Bull. 2001; 27:187-195.

22. N. Mehta, S. Clement, E. Marcus, A.-C. Stona, N. Bezborodovs, S. Evans Lacko, J. Palacios, $\quad$ M. Docherty, E. Barley, D. Rose, M. Koschorke, R. Shidhaye, C. Henderson, G. Thornicroft. Evidence for effective interventions to reduce mental health-related stigma and discrimination in the medium and long term: systematic review. The British Journal of Psychiatry . 2015, 207 (5) 377-384.

23. Weiner, B., Perry, R. P., \&Magnusson, J. (1988). An attribution al analysis of reactions to stigmas. Journal of Personality and Social Psychology. 1988; 55, $738-748$.

24. Reisenzein, R. A structural equation analysis of Weiner's attribution-affect model of helping behavior. Journal of Personality and Social Psychology.1986; $50,1123-1133$. 
25. Muñoz M, Guillén AI, Pérez-Santos E, Corrigan PW. A structural equation modeling study of the Spanish Mental Illness Stigma Attribution Questionnaire (AQ-27-E). Am J Ortho psychiatry. 2015 May; 85(3):243-9.

26. Corrigan, P. W., Rowan, D., Green, A., Lundin, R., River, P., UphoffWasowski, K., Kubiak, M. A. Challenging two mental illness stigmas: Personal responsibility and dangerousness. Schizophrenia Bulletin. 2002; 28, 293-309.

27. Corrigan, PW, Morris, SB, Michaels, PJ, Rafacz, JD, and Rüsch, N. Challenging the public stigma of mental illness: a meta-analysis of outcome studies. Psychiatr Serv. 2012; 63: 963-973.

28. Schachter, HM, Girardi, A, Ly, M et al. Effects of school-based interventions on mental health stigmatization: a systematic review. Child Adolesc Psychiatry Ment Health. 2008; 2: 18.

29. Evans-Lacko, S, Malcolm, E, West, K et al. Influence of Time to Change's social marketing interventions on stigma in England 2009-2011. Br J Psychiatry Suppl. 2013; 55: s77-s88.

30. National Institute of Health and Clinical Excellence. Behaviour change at population, community and individual levels. http://www.nice.org.uk/PH006; 2007.

31. Corrigan, $\mathrm{P}$ and Watson, A. The paradox of self-stigma and mental illness. Clin Psychol Sci Pract.2006; 9: 35-53. 


\section{Título}

Estigma: la relevancia del contacto social en el trastorno mental.

Stigma: the relevance of social contact in mental disorder.

\section{Autores:}

Víctor M. Frías ${ }^{1}$, Joan R. Fortuny ${ }^{2}$, Sergio Guzmán ${ }^{2}$, Pilar Santamaría ${ }^{2}$, Montserrat Martínez ${ }^{3}$, Víctor Pérez ${ }^{4}$.

Lugar de trabajo: ${ }^{1}$ Institut de Neuropsiquiatria i Addicions, Centre Assitencial Dr. Emili Mira, Parc de Salut Mar. Departament de Psiquiatria, Universidad Autonoma de Barcelona. ${ }^{2}$ Institut de Neuropsiquiatria i Addicions, Centre Assitencial Dr. Emili Mira, Parc de Salut Mar. ${ }^{3}$ Gestió del Coneixement i Avaluació Hospital Universitari Vall d'Hebron, Barcelona. ${ }^{4}$ Institut de Neuropsiquiatria i Addiccions, Hospital del Mar, Barcelona. IMIM (Institut Hospital del Mar d'Investigacions Mèdiques), Barcelona.CIBERSAM. Departament de Psiquiatria, Universitat Autònoma de Barcelona.

Correspondencia: Víctor M Frías. Avda. Prat de la Riba 171, Sta. Coloma de Gramenet, 08921 Barcelona.vfriastrigo@parcdesalutmar.cat

\section{Agradecimientos}

Los autores agradecen a R. Pascual y a su equipo su contribución en el estudio del campeonato de futbol, a G. Quero coordinadora del centre cívic del barri llatí y a P. Rodríguez con todo su equipo de coordinación de los centros cívicos del Ayuntamiento de Sta. Coloma de Gramenet. Así como a D. Ávalos y P. Gómez. 
TABLA 1. Análisis de medias sobre la competición de fútbol ( $n=42)$, $t$-student.

\begin{tabular}{lccccc}
\hline Dimensiones & $\begin{array}{c}\text { Pre-contacto } \\
\text { T1 Media (DE) }\end{array}$ & $\begin{array}{c}\text { Post-contacto } \\
\text { T2 Media (DE) }\end{array}$ & $\begin{array}{c}\text { IC = 95\% } \\
\text { Inferior- superior }\end{array}$ & t & P \\
\hline Responsabilidad & $10,17(3,305)$ & $11,02(3.732)$ & $-2,221 ; 0,507$ & $-1,269$ & 0,211 \\
Piedad & $17,43(3,826)$ & $14,71(3,744)$ & 1,$217 ; 4,212$ & 3,661 & $0,001^{*}$ \\
Ira & $7,52(3,730)$ & $6,93(4,447)$ & $-0,927 ; 2,117$ & 0,790 & 0,434 \\
Peligrosidad & $9,31(4,662)$ & $8,29(5,004)$ & $-0,424 ; 2,472$ & 1,428 & 0,161 \\
Miedo & $7,33(4,476)$ & $6,67(4,365)$ & $-0,969 ; 2,302$ & 0,823 & 0,415 \\
Ayuda & $19,52(5,167)$ & $19,57(6,333)$ & $-2,110 ; 2,015$ & $-0,047$ & 0,963 \\
Coacción & $21,24(3,587)$ & $16,9(3,420)$ & 2,$889 ; 5,778$ & 6,057 & $0,000^{*}$ \\
Segregación & $10,9(4,487)$ & $9,95(5,118)$ & $-1,038 ; 2,942$ & 0,967 & 0,339 \\
Evitación & $16,48(6,840)$ & $17,46(6,096)$ & $-3,182 ; 0,611$ & $-1,369$ & 0,178 \\
\hline
\end{tabular}

*Significación, $p=\leq 0,05$

TABLA 2. Análisis de medias sobre la convivencia cotidiana, Población general (PG) $(n=64)$ y SI contacto ( $n=100)$, t-student.

\begin{tabular}{lrrrcc}
\hline \multicolumn{1}{c}{ Dimensiones } & \multicolumn{1}{c}{ PG } & SI contacto & IC=95\% & t & Pedia(DE) \\
& Media (DE) & Inferior- superior & & \\
\hline Responsabilidad & $10,25(3,528)$ & $9,68(4,261)$ & $-0,692 ; 1,832$ & 0,892 & 0,374 \\
Piedad & $14,75(5,195)$ & $17,26(4,788)$ & $-4,075 ;-0,945$ & $-3,168$ & $0,002^{*}$ \\
Ira & $7,48(4,442)$ & $5,75(4,101)$ & 0,$407 ; 3,062$ & 2,580 & $0,011^{*}$ \\
Peligrosidad & $9,47(5,481)$ & $6,47(4,745)$ & 1,$419 ; 4,581$ & 3,746 & $0,000^{*}$ \\
Miedo & $8,67(5,774)$ & $5,99(4,676)$ & 1,$060 ; 4,304$ & 3,265 & $0,001^{*}$ \\
Ayuda & $18,38(6,093)$ & $22,06(5,363)$ & $-5,474 ;-1,896$ & $-4,068$ & $0,000^{*}$ \\
Coacción & $20,31(4,268)$ & $18,41(6,344)$ & 0,$123 ; 3,682$ & 2,112 & $0,036^{*}$ \\
Segregación & $9,77(5,629)$ & $8,07(6,296)$ & $-0,189 ; 3,594$ & 1,777 & 0,077 \\
Evitación & $15,53(7,509)$ & $18,39(6,809)$ & $-5,100 ;-0,618$ & $-2,519$ & $0,013^{*}$ \\
\hline
\end{tabular}

*Significación, $p=\leq 0,05$ 